\title{
Calorimetric study of melted DNA glass
}

\author{
Jessica Valle-Orero*, Jean-Luc Garden ${ }^{\dagger}$, Jacques Richard ${ }^{\dagger}$, Andrew Wildes* and \\ Michel Peyrard**
}

\author{
*Institut Laue Langevin, BP 156, 6, rue Jules Horowitz 38042 Grenoble Cedex 9, France \\ ${ }^{\dagger}$ Institut Néel, CNRS - Université Joseph Fourier, 25 rue des Martyrs, BP 166, 38042 Grenoble cedex 9, France \\ ${ }^{* *}$ Laboratoire de Physique, Ecole Normale Supérieure de Lyon, 46 allée d'Italie, 69364 Lyon Cedex 07, France
}

\begin{abstract}
.
In this work we report a detailed calorimetric study using differential scanning calorimetry (DSC) of the glassy behaviour of films made from DNA fibers. We show that after the first irreversible thermal denaturation of the molecules when the sample is reiteratively heated, it reaches a disordered state in which it presents a typical glass behaviour during successive cooling and heating scans. Furthermore, we have investigated the properties of these DNA films in two series of experiments, a slow cooling at different rates followed by a DSC scan upon heating, and ageing at a temperature below the glass transition. Introducing the fictive temperature to characterise the glass allows us to derive quantitative information on the relaxations of the DNA films, in particular to evaluate their enthalpy barrier. A comparison with similar ageing studies on PVAc highlights some specificities of the DNA samples.
\end{abstract}

Keywords: Enthalpy Relaxation; Structural Relaxation; Deoxyribonucleic Acid; Amorphous Material; Polyvinyl Acetate;Differential Scanning Calorimetry

PACS: $65.60 .+\mathrm{a}$

\section{INTRODUCTION}

The loss of the double helical structure of DNA as a consequence of thermal denaturation has a strong effect of the whole structure of the fibres and films. While DNA is fairly rigid in its double helix form, when the two single strands dissociate after denaturation they become very flexible, and at high temperature this leads to a sample in which the strands are fully disordered and entangled, in a quasi-liquid state.

A glass transition is a transformation in which an amorphous system on cooling undergoes a transformation from liquid (equilibrium) to glass (non-equilibrium) without being able to crystalise. As the temperature of an homogeneous vitreous system in a liquid state is lowered their inter and intramolecular motions become too slow to be detectable experimentally. The liquid is impeded from reaching equilibrium which can only be attained by structural relaxation or ageing at very long timescales.

For DNA films, even though the stacking of the base pairs is broken, the intermolecular confinement is still present and influences the relaxations in the glassy state. During ageing or slow cooling the system evolves towards states with lower and lower enthalpy. Conversely, when the system is heated up after ageing, it can absorb the corresponding enthalpy at high temperature, which shows up as a peak in the heat capacity. The rate of temperature variation defines the timescales at which the system is allowed to change. The dependence of the glass structure on the cooling rate can be described by the lim- iting fictive temperature, $T_{f}^{\prime}[1]$.

The glass character of DNA has been studied previously. Tsereteli's group [2] presented many published reports on the glass character of DNA in solution. Observations of the glassy behaviour in fibres were made [3] but the relaxation character of these glass-like systems was never quantitatively analysed.

In this paper, we report a detailed calorimetric study of the glassy behaviour of melted films made from DNA fibres. We compare glassy DNA melt properties with PVAc, a classical polymeric glass former. The same experimental protocols (different cooling and ageing times) are used to assess the glassy properties of the two systems. For ageing experiments, we use two different approaches concerning the evolution of the relaxation times, which lead to the same conclusion. One is based on the Tool-Narayanaswamy-Moynihan (TNM) [1] expression of the relaxation time, while the other uses an Arrhenius expression. For both approaches, the concept of limiting fictive temperature is used. It is however not usual to calculate limiting fictive temperatures after ageing experiments, it is why we focalise on this point in this paper. 


\section{EXPERIMENTAL SECTION}

\section{Sample Preparation}

The samples consisted of Oriented Fibres of DNA prepared using the "wet spinning technique" which has been meticulously described elsewhere [4]. Once the oriented fibres are prepared they go through a process of cleaning, drying and humidification. For the purpose of our experiments the films were humidified to $56 \% \mathrm{RH}$ for several weeks.

A Setaram Micro DSC III was used to perform the measurements. For each of the DSC experiments one or several hydrated films with a mass between 80 and 100 $\mathrm{mg}$ were rolled up and sealed inside an hastelloy tube. The sample was then placed in the DSC at $293 \mathrm{~K}$ and left for 10 minutes for stabilisation. For the reference tube, $345 \mathrm{mg}$ of VESPEL was used, as it does not exhibit a glass transition within the region of our study.

\section{Experimental Protocol}

During the first raise in temperature from $268 \mathrm{~K}$ to 368 $\mathrm{K}$ at a rate of $1.2 \mathrm{~K} / \mathrm{min}$, the sample goes through a denaturing transition as it is observed in DNA solution. It was then cooled down to $268 \mathrm{~K}$ at the same rate. The cooling scan showed a glass transition at around $298 \mathrm{~K}$. The sample was kept inside the DSC throughout the different measurements. Before each experiment, the sample was exposed to a series of cooling-heating temperature cycles and finally heated to $363 \mathrm{~K}$ during $20 \mathrm{mins}$, this is referred as "pre-experiment". This was done to erase any annealing thermal history. The data presented in specific heat $\triangle C$ as a function of temperature were first corrected from instrumental resolution and then the VESPEL contribution was isolated in order to obtain only the heat capacity of DNA. The resulting data and the protocol have been presented in detail in a recent publication [5].

Two series of experiments were carried out with the same sample to study the structural relaxation behaviour of the glassy system obtained by heating a film of oriented DNA fibers up to denaturation temperature and cooling it down to room temperature.

1. Different cooling rates. This consisted of a succession of temperature ramps. Starting from the initial state at $363 \mathrm{~K}$, the sample was cooled down at a rate $\beta$ from 363 to $268 \mathrm{~K}$; left for 5 mins for equilibration; and heated to $363 \mathrm{~K}$ at $1.2 \mathrm{~K} / \mathrm{min}$. It was then stabilised for $20 \mathrm{~min}$ at this highest temperature before the next cooling ramp, which was recorded as a DSC scan. The cooling rate was modified at each ramp in the following order, $\beta=1.2,0.4,0.2,0.1$, $0.05,0.033,0.01$ and back to $1.2 \mathrm{~K} / \mathrm{min}$. The heat- ing rate was kept fixed.

2. Annealing at different ageing times. The procedure consisted in cooling at $1.2 \mathrm{~K} / \mathrm{min}$ from $363 \mathrm{~K}$ to the ageing temperature $T_{a}$ below the glass transition temperature, annealing the sample for a period of time $t_{a}$, and then cooling down to $268 \mathrm{~K}$ before recording the DSC signal on heating from $268 \mathrm{~K}$ to $363 \mathrm{~K}$. All temperature ramps were programmed at a rate of $1.2 \mathrm{~K} / \mathrm{min}$. The relaxation of the system was studied at fifteen different ageing times, $t_{a}=84$, $78,72,66,60,54,48,42,32,20,10,6,3,1$ and 0 hours. The last corresponds to an experiment without annealing. However by simply taking into account the minimal response time of the calorimeter one can consider that the effective minimal ageing time is actually equal to $10 \mathrm{~min}$.

\section{RESULTS}

\section{Cooling at different temperature rates}

An enthalpic response was obtained for each of the DSC heating ramps at $1.2 \mathrm{~K} / \mathrm{min}$ after cooling at the different rates $\beta$. The data expressed as normalised heat capacity as a function of temperature was published elsewhere [5]. For each of the normalised curves the limiting fictive temperature $T_{f}^{\prime}$ was calculated using Equation 1 given by Moynihan [1]. The same experimental procedure and analysis was carried out for PVAc.

$$
\int_{T^{*}}^{T_{l}}\left(C p-C p_{g}\right) d T=\int_{T_{f}^{\prime}}^{T_{l}}\left(C p_{l}-C p_{g}\right) d T,
$$

where $C p$ is the specific heat, $C p_{g}$ and $C p_{l}$ are the specific heat for the glassy and liquid state respectively, and $T_{l}$ and $T^{*}$ are arbitrary temperatures in the liquid and glass region further away from the transition region.

The Arrhenius law,

$$
\tau \propto \frac{1}{\beta} \propto \exp \left(\frac{\triangle h}{T_{f}^{\prime}}\right),
$$

was applied to the data in order to obtain the activation energy $\triangle h$ in units of temperature (in $K: \triangle h=\triangle H^{*} / R$, where $\triangle H^{*}$ is the activation enthalpy in $\mathrm{J} / \mathrm{mol}$, and $R$ is the ideal gas constant in $\mathrm{J} /(\mathrm{mol} \cdot \mathrm{K})$ for both systems. These gave activation energies of $79 \mathrm{kK}$ and $99 \mathrm{kK}$ for DNA glass and PVAc respectively. These values are of the same order of magnitude. See Table 1. 


\section{Annealing at different ageing times}

The samples were annealed at a temperature $T_{a}$ which is $15 \mathrm{~K}$ below the inflection point of their respective specific heat curve measured during the cooling run at $1.2 \mathrm{~K} / \mathrm{min}$, i.e., $T_{a, D N A}=293 \mathrm{~K}$ and $T_{a, P V A c}=297 \mathrm{~K}$ for DNA and PVAc. As stated before, we investigated a series of ageing times (relaxing times) $t_{a}$ varying from 84 $\mathrm{h}$ to $1 \mathrm{~h}$, and even no ageing at all. In our previous work we proved that both samples behave in a qualitatively similar way [5]. However, for PVAc a peak is visible during reheating even without ageing, while for DNA there is no peak in the absence of ageing. This suggests that underlying relaxation mechanisms for the two glassformers do not obey the same laws.

The DSC heating scans for melted DNA annealed with different annealing times $t_{a}$, as described in the experimental method, showed a progressive growth of an endothermic peak with the increase of $t_{a}$ [5]. The first step in understanding the state of the sample after annealing is to calculate the area under the endothermic peak, also called "enthalpy recovery" $\triangle H$. This property corresponds to an enthalpy decrease during the annealing process, which is again absorbed by the system to reach the equilibrium state at high temperature. It characterises the annealed glassy state from the energetic viewpoint. Furthermore, the annealed system can also be characterised by another property, its limiting fictive temperature, the same way we used it in the previous section to characterise glassy states obtained at different cooling rates. Owing to our experimental protocol in which we freeze the system to low temperature $(268 \mathrm{~K})$ before recording the DSC signal with increasing temperature, we apply Equation 1 to the specific heat curves. This provides a value of $T_{f}^{\prime}$, which describes the instantaneous state obtained after ageing that has been frozen at lower temperature.

Once this was calculated, the data were analysed using two different expressions of the relaxation time. In both cases, the limiting fictive temperature was used for the characterisation of ageing experiments. The first method consists in a treatment based on the TNM expression of the relaxation time, in which a type of ageing time shift factor $R\left(T_{a}, t_{a}\right)$ is used to compare the evolution during ageing of the typical relaxation times $t_{a}$, between DNA and PVAc, at the ageing temperature $T_{a}[6]$. The second treatment considers an Arrhenius law for the expression of the relaxation time, in which contrary to the former method, the ageing time shift factor is defined differently in order to follow the evolution of the relaxation times on the equilibrium liquid line for the two systems. Both treatments will be explained further in the following sections.

Figure 1 gives a graphical description of the concept of fictive temperature $T_{f}$ as a function of temperature.

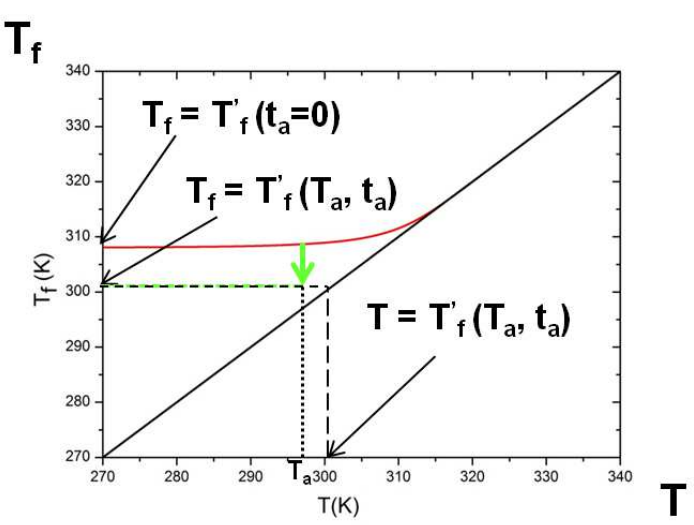

FIGURE 1. Evolution of the fictive temperature versus temperature after annealing; see text for details.

The graph shows how after annealing, the limiting fictive temperatures can be obtained. The continuous red curve corresponds to a cooling ramp at $1.2 \mathrm{~K} / \mathrm{min}$, where the ending point of the cooling measurement is $T_{f}=T_{f}^{\prime}\left(t_{a}=\right.$ $0)$, when no ageing is applied. The two characterisations of the ageing described above have been represented, the differences lie within the ending point. In the first method, the system is cooled down at the same rate, annealed at $T_{a}$ (marked by the arrow), and finally cooled down to a temperature lower than $T_{a}$; the relaxation time is therefore calculated at the ageing temperature $\tau\left(T_{a}, t_{a}\right)$ where the fictive temperature at that position $T_{f}$ can be assumed to equal $T_{f}^{\prime}\left(T_{a}, t_{a}\right)$. In the second treatment, the experimental method is the same but the relaxation time is considered to be a function of the limiting fictive temperature, where $T=T_{f}^{\prime}\left(T_{a}, t_{a}\right)$.

\section{First treatment: Ratio of the relaxation times at the ageing temperature}

In order to characterise the behaviour of a glass former after ageing experiments, it is usual to represent the ageing time shift factor $a_{t_{a}}=\tau\left(T_{a}, t_{a}\right) / \tau\left(T_{a}, t_{a, r}\right)$ as a function of the ageing time [6]. Where $\tau\left(T_{a}, t_{a}\right)$ is the relaxation time at the ageing temperature $T_{a}$ and at the ageing time $t_{a}$, and $t_{a, r}$ is a reference ageing time. Here we present the same type of ratio using the TNM expression of the typical relaxation time:

$$
\tau=\tau_{0} \exp \left(x \frac{\triangle h}{T}+(1-x) \frac{\triangle h}{T_{f}}\right) .
$$

In this expression, $\tau_{0}$ is related to the typical relaxation time of molecular vibrations, $\triangle h$ is the activation enthalpy obtained by means of the different cooling rates experiments, $T$ and $T_{f}$ are the temperature and the fictive 
temperature respectively, and $x$ is the non-linearity parameter pertaining to the glass structure dependence of the typical relaxation time. In this case, the ageing time shift factor could be written as:

$$
R\left(T_{a}, t_{a}\right)=\frac{\tau_{0} \exp \left(x \frac{\triangle h}{T_{a}}+(1-x) \frac{\triangle h}{T_{f}}\right)}{\tau_{0} \exp \left(\frac{\triangle h}{T_{f}^{\prime}}\right)} .
$$

The fictive temperature appearing in the numerator is a function of the ageing temperature and the ageing time $T_{f}=T_{f}\left(T_{a}, t_{a}\right)$. From here onwards, we will assume that this fictive temperature is the same that the limiting fictive temperature calculated using Equation 1. This assumption is valid because just after the ageing time the system is cooled again to the lowest temperature before reheating. Therefore, we suppose that during this second cooling the fictive temperature of the system had no time to relax and remained in the state reached after ageing at $T_{a}$. This assumption becomes more accurate as the ageing time increases. Indeed, for long ageing time the real fictive temperature of the system at $T_{a}$ does not have time to evolve significantly within the time interval of the second cooling. The reference relaxation time in the denominator was defined by using the limiting fictive temperature $T_{f}^{\prime}$ calculated for the DSC heating ramp obtained after cooling at $1,2 \mathrm{~K} / \mathrm{min}$. In other words, the reference ageing time is chosen for an experiment without ageing or for a reference ageing time $t_{a, r}$ equal to zero. The non-linearity parameter $x$ is calculated by means of the Kovacs-Aklonis-Hutchinson-Ramos (KAHR) approach [7]. From each of the DSC heating curves obtained at the different ageing times, the enthalpy recovery $(\triangle H)$ and the temperature of the maximum of the peak $T_{p}$ were determined. Figure 2 shows $T_{p}$ as a function of $\triangle H$ for each ageing time.

The outside-saturation range shows a straight line with a rate $d T_{p} / d(\triangle H)$ of about $4.03 \mathrm{gK} / \mathrm{J}$ and $1.8 \mathrm{gK} / \mathrm{J}$ for DNA and PVAc respectively. From the measurements carried out at the reference cooling rate $\beta=1.2 \mathrm{~K} / \mathrm{min}$, the step-like descent exhibited by the specific heat at the glass temperature $\triangle C_{p}\left(T=T_{g}\right)$ was estimated to 0.505 $\mathrm{J} / \mathrm{gK}$ for DNA and $0.52 \mathrm{~J} / \mathrm{gK}$ for PVAc. Henceforth, using KHAR's method the parameter $x$ was calculated to 0.3 for DNA and 0.48 for PVAc. Table 1 gives a summary of the characteristic values defined using the first method for both DNA and PVAc.

The data for DNA glass and PVAc was analysed using the first treatment for the ratio of the relaxation times at the ageing temperature $t_{a}$. Figure 3 shows the ageing time shift factor determined as a function of the ageing temperature.

While the ageing time shift factor seems to behave similarly for DNA and PVAc for the points comprised within the 33 first hours of measurements (with only a difference in amplitude), a clear change occurs in the variation rate for the longest ageing times. The factor seems to undergo a saturation for PVAc while for DNA it increases dramatically.

\section{Second treatment: Ratio of the relaxation times at different limiting fictive temperatures}

A different analysis was carried out with the same data for both systems [5]. This differs from the one described above as it is based on a different expression for the relaxation time. It aims, this time, to study the ageing dependence of the relaxation times at $T_{f}^{\prime}$, and thus to define the ageing time shift factor on the basis of the Arrhenius law. Indeed, by definition, the non-equilibrium state of a frozen system at a given temperature (in our case $T_{a}$ ) can be described by the corresponding equilibrium state (liquid), but taken at the temperature defined by the limiting fictive temperature. Making $T_{f}=T_{f}^{\prime}$ and $T=T_{f}^{\prime}$ in the TNM expression of the relaxation time leads to an Arrhenius law. As already said, the state of the sample after annealing can also be characterised by the limiting fictive temperature. This is also consistent with the previous assumption that the fictive temperature after ageing can be frozen by the second cooling at the lowest temperature. Within this scenario, a type of ageing time shift factor can be defined such as follows:

$R^{\prime}\left(T_{a}, t_{a}\right)=\frac{\tau_{0} \exp \left(\frac{\triangle h}{T_{f}^{\prime}\left(T_{a}, t_{a}\right)}\right)}{\tau_{0} \exp \left(\frac{\triangle h}{T_{f}^{\prime}\left(t_{a}=0\right)}\right)}=\exp \left(\frac{\Delta h}{T_{f}^{\prime}\left(T_{a}, t_{a}\right)-T_{f}^{\prime}\left(t_{a}=0\right)}\right)$.

Where $T_{f}^{\prime}\left(t_{a}=0\right)$ is the limiting fictive temperature calculated for the reference cooling experiment with a temperature rate of $1,2 \mathrm{~K} / \mathrm{min}$ (the same than for the previous treatment).

Figure 4 shows the ageing time shift factor $R^{\prime}$ determined as a function of the limiting fictive temperature.

In this treatment, one has to bear in mind that the evolution of the relaxation time is characteristic of the corresponding equilibrium liquid taken at different decreasing temperatures (limiting fictive temperatures), the temperature limit being the real temperature of ageing of the system. The major advantage of such a treatment is that it does not involve a complex phenomenological KAHR model.

As one can notice the ratios $R$ and $R^{\prime}$ are different, but this is not surprising since they measure different quantities. $R$ takes into account the KHAR's parameter $x$, while $R^{\prime}$ does not. And thus, the differences are not due to experimental errors. It is difficult to give a physical meaning to the absolute values of these numbers but what 

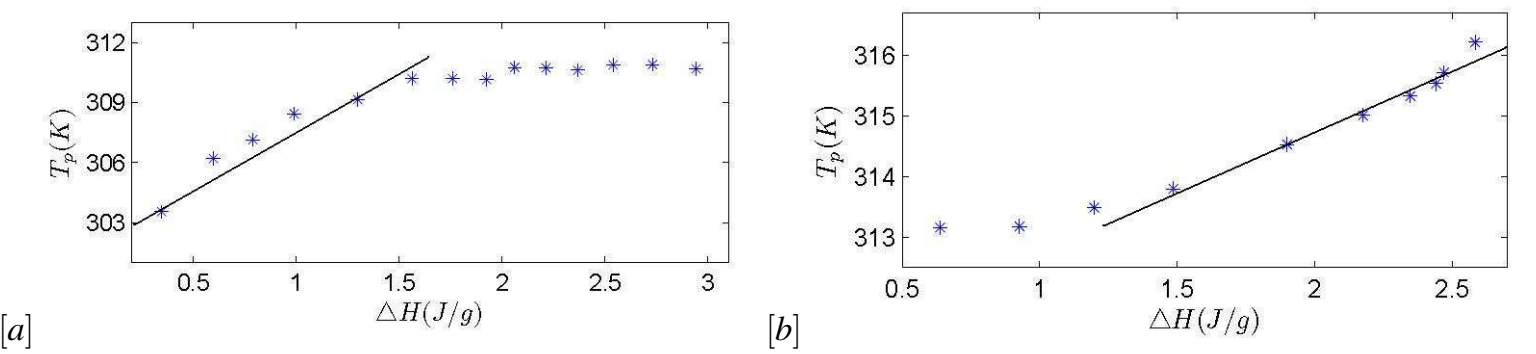

FIGURE 2. Dependence of the recovery enthalpy $\triangle H$ on the temperature of the enthalpic peak $T_{p}$ for DNA (a) and PVAc (b). The straight lines corresponds to linear fits of the measurements outside the saturation range, which slope was estimated to 4.03 $\mathrm{gK} / \mathrm{J}$ and $1.8 \mathrm{gK} / \mathrm{J}$ for DNA and PVAc respectively

TABLE 1. Summary of the calculated values relevant for the analysis of the relaxation behaviour of both systems, DNA and PVAc. The first two, $\frac{d T_{p}}{\Delta H}$ and $\triangle C_{p}$, were necessary in order to obtain the non-linearity parameter $x$.

\begin{tabular}{|l|c|c|c|c|c|c|c|}
\hline & $T_{a}(\mathrm{~K})$ & $T_{g}(\mathrm{~K})$ & $\frac{d T_{p}}{d \triangle H}(\mathrm{gK} / \mathrm{J})$ & $\triangle C_{p}(\mathrm{~J} / \mathrm{gK})$ & $\mathrm{x}$ & $\triangle h(\mathrm{kK})$ & Molecular weight \\
\hline DNA & 293 & 308 & 4.03 & 0.51 & 0.30 & 79 & $1.3 \times 10^{7}$ \\
PVAc & 297 & 312 & 1.80 & 0.52 & 0.48 & 99 & $1.6 \times 10^{5}$ \\
\hline
\end{tabular}

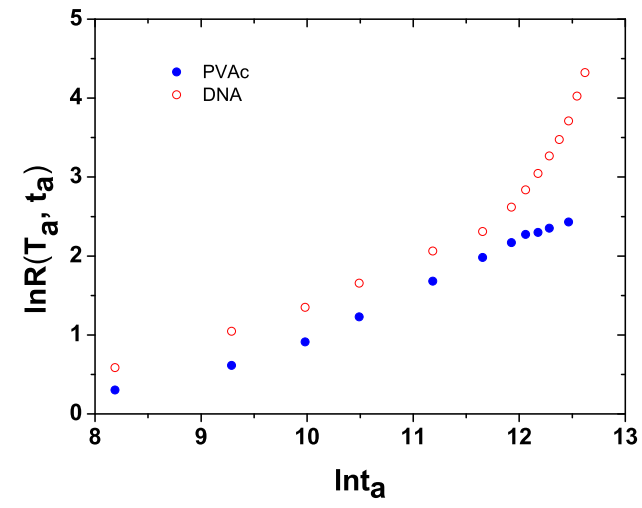

FIGURE 3. Evolution of the ageing time shift factor $R\left(T_{a}, t_{a}\right)$ versus the logarithm of the ageing time $\ln \left(t_{a}\right)$ for DNA (open circles) and PVAc (filled circles). The data was analysed using the first treatment, in which the relaxation time is defined at the ageing temperature.

it is important is their evolution with ageing time. Both figures show that for PVAc $R$ and $R^{\prime}$ vary almost linearly with $\ln \left(t_{a}\right)$, while for DNA glass there is a significant raise of the two ratios for long ageing times. We come back to this point in the discussion section.

\section{DISCUSSION}

Our calorimetric study of a DNA film has shown that, after the thermal denaturation of the double helix, this

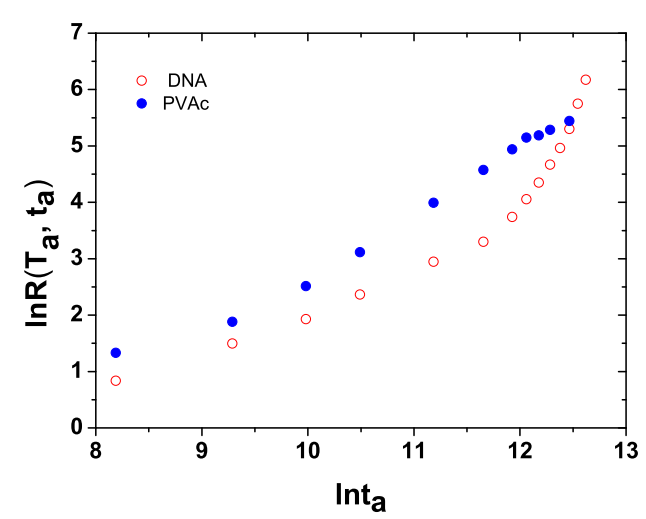

FIGURE 4. Evolution of the ageing time shift factor $R^{\prime}\left(T_{a}, t_{a}\right)$ versus the logarithm of the ageing time $\ln \left(t_{a}\right)$ for DNA (open circles) and PVAc (filled circles). The data was analysed using the second treatment, in which the relaxation time is defined at the limiting fictive temperature $T_{f}^{\prime}$.

system has properties which are very similar to those of a polymer glass, such as PVAc, with however noticeable differences

1. The activation energies for DNA $(80 \mathrm{kK})$ and PVAc $(100 \mathrm{kK})$ are slightly different, but are on the same order of magnitude.

2. While DNA glass and PVAc seem to behave similarly for short ageing times at $T_{g}-15 \mathrm{~K}$, they behave differently for ageing times larger than about 30 hours. The increase of the relaxation time for 
DNA seems to indicate that ageing occurs in two stages. In a first stage the system can relax rapidly to finally reach a state in which relaxation becomes very slow in the second stage. This second stage only concerns very long ageing times.

The activation energies $\triangle h$ for PVAc and DNA glass are measuring the energies needed to break the chemical bonds to allow the motion of the systems. For both, PVAc and DNA glass, these are weak bonds between organic elements, and thus from an energetic point of view these two compounds are not very different. This is the reason why we measure comparable activation energies (point 1). On the contrary, in point 2 , we show that DNA glass relaxes slower at longer ageing times than PVAc. This difference in the relaxation times could be related to kinetic effects. For DNA, the sliding of the chains is hindered by their particular structure: they have bases dangling on the side, so that, due to the confinement of the environment, the large amplitude motions of the structure as a whole will certainly be a very slow process.

From a methodology point of view, we have shown that it is relevant to describe the ageing character of a system by the limiting fictive temperature $T_{f}^{\prime}$ and hence quantify the relaxation time as a function of this property $\tau\left(T_{f}^{\prime}\right)$.

Similar experiments at different ageing temperatures in the glassy region could complete this study. This could bring insights into the time evolution of the typical relaxation times as a function of temperature for PVAc and DNA glass.

\section{REFERENCES}

1. C. T. Moynihan, P. B. Macedo, C. J. Montrose, P. K. Gupta, M. Debolt, J. Dill, B. Dom, P. Drake, A. Easteal, P. Elterman, R. Moeller, H. Sasabe, and J. Wilder, Annals of the New York Academy of Sciences 279, 15-35 (1976).

2. G. I. Tsereteli, T. Belopolskaya, N. Grunina, and O. Vaveliouk, Journal of Thermal Analysis and Calorimetry 62, 89-99 (2000).

3. S. Lee, P. Debenedetti, J. Errington, B. Pethica, and D. Moore, J. Phys. Chem. B 108, 3098-3106 (2004).

4. A. Rupprecht, Biochimica et Biophysica Acta 199, 277-280 (1970).

5. J. Valle-Orero, J.-L. Garden, J. Richard, A. Wildes, and M. Peyrard, Journal of Physical Chemistry B 116 4394-4402 (2012).

6. J. Zhao, and G. B. McKenna, J. Chem. Phys 136, 154901 (2012).

7. J. M. Hutchinson, and P. Kumar, Thermochimica. Acta. 391, 197-217 (2002). 